\title{
IGUSA AND DENEF-SPERBER CONJECTURES ON NONDEGENERATE $p$-ADIC EXPONENTIAL SUMS
}

\author{
by
}

\author{
Raf Cluckers
}

\begin{abstract}
We prove the intersection of Igusa's Conjecture of [Igusa, J., Lectures on forms of higher degree, Lect. math. phys., Springer-Verlag, 59 (1978)] and the Denef - Sperber Conjecture of [Denef, J. and Sperber, S., Exponential sums mod $p^{n}$ and Newton polyhedra, Bull. Belg. Math. Soc., suppl. (2001) 55-63] on nondegenerate exponential sums modulo $p^{m}$.
\end{abstract}

\section{Introduction}

Let $f$ be a polynomial over $\mathbf{Z}$ in $n$ variables. Consider the "global" exponential sum

$$
S_{f}(N):=\frac{1}{N^{n}} \sum_{x \in\{0, \ldots, N-1\}^{n}} \exp \left(2 \pi i \frac{f(x)}{N}\right),
$$

where $N$ varies over the positive integers. In order to bound $\left|S_{f}(N)\right|$ in terms of $N$, it is enough to bound

$$
\left|S_{f}\left(p^{m}\right)\right|
$$

in terms of $m>0$ and prime numbers $p$. When $f$ is nondegenerate in several senses related to its Newton polyhedron, specific bounds which depend uniformly on $m$ and $p$ have been conjectured by Igusa and by Denef - Sperber.

We prove these bounds, thus solving a conjecture by Denef and Sperber from a 1990 manuscript 7] (published in 2001 [8]), and the nondegenerate case of Igusa's conjecture for exponential sums from the introduction of his book [10].

One of the main points of this article is that, while for finite field exponential sums like $S_{f}(p)$ one knows that the weights and Betti numbers have some uniform behaviour for big $p$, for $p$-adic exponential sums $S_{f}\left(p^{m}\right)$ one does not yet completely

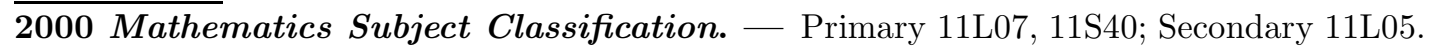

${ }^{*}$ During the realization of this project, the author was a postdoctoral fellow of the Fund for Scientific Research - Flanders (Belgium) (F.W.O.) and was supported by The European Commission - Marie Curie European Individual Fellowship with contract number HPMF CT 2005-007121. 
know what the analogues of the weights and Betti numbers are, let alone that they have some uniform behaviour in $p$.

Notation. - Let $f$ be a nonconstant polynomial over $\mathbf{Z}$ in $n$ variables with $f(0)=$ 0 . Write $f(x)=\sum_{i \in \mathbf{N}^{n}} a_{i} x^{i}$ with $a_{i} \in \mathbf{Z}$. The global Newton polyhedron $\Delta_{\text {global }}(f)$ of $f$ is the convex hull of the support $\operatorname{Supp}(f)$ of $f$, with

$$
\operatorname{Supp}(f):=\left\{i \mid i \in \mathbf{N}^{n}, a_{i} \neq 0\right\} .
$$

The Newton polyhedron $\Delta_{0}(f)$ of $f$ at the origin is

$$
\Delta_{0}(f):=\Delta_{\text {global }}(f)+\mathbf{R}_{+}^{n}
$$

with $\mathbf{R}_{+}=\{x \in \mathbf{R} \mid x \geq 0\}$ and $A+B=\{a+b \mid a \in A, b \in B\}$ for $A, B \subset \mathbf{R}^{n}$. For a subset $I$ of $\mathbf{R}^{n}$ define

$$
f_{I}(x):=\sum_{i \in I \cap \mathbf{N}^{n}} a_{i} x^{i}
$$

By the faces of $I$ we mean $I$ itself and each nonempty convex set of the form

$$
\{x \in I \mid L(x)=0\}
$$

where $L(x)=a_{0}+\sum_{i=1}^{n} a_{i} x_{i}$ with $a_{i} \in \mathbf{R}$ is such that $L(x) \geq 0$ for each $x \in I$. By the proper faces of $I$ we mean the faces of $I$ that are different from $I$. For $\mathcal{I}$ a collection of subsets of $\mathbf{R}^{n}$, call $f$ nondegenerate with respect to $\mathcal{I}$ when $f_{I}$ has no critical points on $\left(\mathbf{C}^{\times}\right)^{n}$ for each $I$ in $\mathcal{I}$, where $\mathbf{C}^{\times}=\mathbf{C} \backslash\{0\}$. For $k \in \mathbf{R}_{+}^{n}$ put

$$
\begin{aligned}
\nu(k) & =k_{1}+k_{2}+\ldots+k_{n}, \\
N(f)(k) & =\min _{i \in \Delta_{0}(f)} k \cdot i, \\
F(f)(k) & =\left\{i \in \Delta_{0}(f) \mid k \cdot i=N(f)(k)\right\},
\end{aligned}
$$

where $k \cdot i$ is the standard inproduct on $\mathbf{R}^{n}$. Denote by $F_{0}(f)$ the smallest face of $\Delta_{0}(f)$ which has nonempty intersection with the diagonal $\{(t, \ldots, t) \mid t \in \mathbf{R}\}$ and let $(1 / \sigma(f), \ldots, 1 / \sigma(f))$ be the intersection point. If there is no confusion, we write $\sigma$ instead of $\sigma(f), N(k)$ instead of $N(f)(k)$, and $F(k)$ for $F(f)(k)$. Let $\kappa$ be the codimension of $F_{0}(f)$ in $\mathbf{R}^{n}$.

\section{The main results}

From here up to section 9 , let $f$ be a nonconstant polynomial over $\mathbf{Z}$ in $n$ variables with $f(0)=0$ (the adaptation to the case $f(0) \neq 0$ is easy).

2.1. Theorem. - Suppose that $f$ is homogeneous and nondegenerate w.r.t. the faces of $\Delta_{0}(f)$. Then there exists $c>0$ and $M>0$ such that

$$
\left|S_{f}\left(p^{m}\right)\right| \leq c p^{-\sigma m} m^{\kappa-1}
$$

$\overline{{ }^{(1)} \text { When } f(0)} \neq 0$, then there is no harm in replacing $f$ by $f-f(0)$ : all corresponding changes in the paper are easily made. 
for all primes $p>M$ and all integers $m>0$, with $\sigma=\sigma(f)$ and $\kappa$ as in the section on notation. Moreover, $c$ can be taken depending on $\Delta_{0}(f)$ only.

One sees that the dependence on $p$ and $m$ is very simple. Since moreover for each $p$ there exists $c_{p}>0$ such that for all $m>0$

$$
\left|S_{f}\left(p^{m}\right)\right| \leq c_{p} p^{-\sigma m} m^{n-1},
$$

by [10], [9], or [3], and properties of toric resolutions, and since $\kappa \leq n$ one finds the following.

2.2. Corollary. - With $f$ as in Theorem 2.1 there exists $c>0$ such that for all primes $p$ and all integers $m>0$,

$$
\left|S_{f}\left(p^{m}\right)\right| \leq c p^{-\sigma m} m^{n-1}
$$

Denef and Sperber [8] prove Theorem 2.1 under the extra condition that no vertex of $F_{0}(f)$ belongs to $\{0,1\}^{n}$. Corollary 2.2 is the nondegenerate case of Igusa's conjecture [10 on exponential sums for toric resolutions of $f$. The exponent $\sigma$ in the bounds of Theorem 2.1 is conjectured to be optimal for infinitely many $p$ and $m$ by Denef and Sargos [5], [6], in analogy to conjectures on the real case. When no vertex of $F_{0}(f)$ belongs to $\{0,1,2\}^{n}$, the bounds in Theorem 2.1 are shown to be optimal for infinitely many $p$ and $m$ in [8], Theorem (1.3).

\section{Denef - Sperber Formula for $S_{f}\left(p^{m}\right)$ for big $p$}

The following proposition has the same proof as Proposition (2.1) of [8] , but is slightly more general. We give the proof for the convenience of the reader.

3.1. Proposition. - Suppose that $f$ is nondegenerate w.r.t. (all) the faces of $\Delta_{0}(f)$. Then there exists $M>0$ such that

$$
S_{f}\left(p^{m}\right)=\left(1-p^{-1}\right)^{n} \sum_{\tau \text { face of } \Delta_{0}(f)}\left(A(p, m, \tau)+E\left(p, f_{\tau}\right) B(p, m, \tau)\right)
$$

for all primes $p>M$ and all integers $m>0$, with

$$
\begin{array}{cl}
A(p, m, \tau):=\sum_{\substack{k \in \mathbf{N}^{n} \\
F(f)(k)=\tau \\
N(f)(k) \geq m}} p^{-\nu(k)}, \\
B(p, m, \tau):=\sum_{\substack{k \in \mathbf{N}^{n} \\
F(f)(k)=\tau \\
N(f)(k)=m-1}} p^{-\nu(k),}
\end{array}
$$


and

$$
E\left(p, f_{\tau}\right):=\frac{1}{(p-1)^{n}} \sum_{x \in\{1, \ldots, p-1\}^{n}} \exp \left(\frac{2 \pi i}{p} f_{\tau}(x)\right)
$$

Proof. - Writing

$$
S_{f}\left(p^{m}\right)=\int_{\mathbf{z}_{p}} \exp \left(\frac{2 \pi i}{p^{m}} f(x)\right)|d x|,
$$

with $|d x|$ the normalized Haar measure on $\mathbf{Q}_{p}^{n}$, we deduce

$$
S_{f}\left(p^{m}\right)=\sum_{\tau \text { face of } \Delta_{0}(f)} \sum_{\substack{k \in \mathbf{N}^{n} \\ F(f)(k)=\tau}} \int_{\text {ord } x=k} \exp \left(\frac{2 \pi i}{p^{m}} f(x)\right)|d x| .
$$

Put $x_{j}=p^{k_{j}} u_{j}$ for $k \in \mathbf{N}^{n}$. Then $|d x|=p^{-\nu(k)}|d u|$ and

$$
f(x)=p^{N(k)}\left(f_{F(k)}(u)+p(\ldots)\right),
$$

where the dots take values in $\mathbf{Z}_{p}$ and where $N(k)=N(f)(k)$. Hence,

$$
S_{f}\left(p^{m}\right)=\sum_{\tau \text { face of } \Delta_{0}(f)} \sum_{\substack{k \in \mathbf{N}^{n} \\ F(f)(k)=\tau}} p^{-\nu(k)} \int_{u \in\left(\mathbf{Z}_{p}^{\times}\right)^{n}} \exp \left(\frac{2 \pi i}{p^{m-N(k)}}\left(f_{\tau}(u)+p(\ldots)\right)\right)|d u|,
$$

where $\mathbf{Z}_{p}^{\times}$denotes the group of $p$-adic units. Because of the nondegeneratedness assumptions, for $\tau$ a face of $\Delta_{0}(f)$ and $p$ a big enough prime, the reduction $f_{\tau} \bmod p$ has no critical points on $\left(\mathbf{F}_{p}^{\times}\right)^{n}$. Hence, the integral in (3.1.3) is zero whenever $m-N(f)(k) \geq 2$. When $m-N(k) \leq 0$, the integral over $\left(\mathbf{Z}_{p}^{\times}\right)^{n}$ in (3.1.3) is just the measure of $\left(\mathbf{Z}_{p}^{\times}\right)^{n}$ and thus equals $\left(1-p^{-1}\right)^{n}$. When $m-N(k)=1$ the integral over $\left(\mathbf{Z}_{p}^{\times}\right)^{n}$ in $(3.1 .3)$ equals $p^{-n}(p-1)^{n} E\left(p, f_{\tau}\right)$. The Proposition now follows from (3.1.3).

\section{Lower bounds for $\nu(k)$}

The main result of this section is:

4.1. Theorem. - Let $\tau$ be a face of $\Delta_{0}(f)$. Then one has for all $k$ with $F(f)(k)=$ $\tau$ that

$$
\nu(k) \geq \sigma(f)(N(f)(k)+1)-\sigma\left(f_{\tau}\right),
$$

where $\sigma(f)$ and $\sigma\left(f_{\tau}\right)$, as well as $\nu(k)$ and $N(f)(k)$ are as in the section on notation.

The main points are that one subtracts $\sigma\left(f_{\tau}\right)$ instead of $\sigma(f)$ and that $\sigma\left(f_{\tau}\right) \leq$ $\sigma(f)$. Subtracting $\sigma(f)$ would yield trivial bounds since one has $\nu(k) \geq \sigma(f) N(k)$ for all $k \in \mathbf{R}_{+}^{n}$. The Theorem's proof is based on two facts: 
4.2. Lemma. - Let $\tau$ be a face of $\Delta_{0}(f)$, and let $R_{j} \in \mathbf{R}^{n}$ be finitely many points belonging to $\tau$. Let $\beta_{j} \geq 0$ satisfy

$$
\sum_{j} \beta_{j} R_{j} \leq(1 / \sigma, \ldots, 1 / \sigma)
$$

where $a \leq b$ for $a, b \in \mathbf{R}^{n}$ means $a_{i} \leq b_{i}$ for all $i$. Then

$$
\sum \beta_{j} \leq 1 \text {. }
$$

Proof. - Clearly there is no point $S$ in the interior of $\Delta_{0}(f)$ that satisfies $S \leq$ $(1 / \sigma, \ldots, 1 / \sigma)$. When $\sum_{j} \beta_{j}>1$, then $\sum_{j} \beta_{j} R_{j}$ lies in the interior of $\Delta_{0}(f)$.

4.3. Corollary. - Let $\tau, R_{j}$, and $\beta_{j}$ be as in Lemma 4.2. Then

$$
\sum \beta_{j} \leq \frac{\sigma\left(f_{\tau}\right)}{\sigma} .
$$

Proof. - Since $\sum_{j} \beta_{j} R_{j} \leq(1 / \sigma, \ldots, 1 / \sigma)$ one has

$$
\frac{\sigma}{\sigma\left(f_{\tau}\right)} \sum_{j} \beta_{j} R_{j} \leq\left(1 / \sigma\left(f_{\tau}\right), \ldots, 1 / \sigma\left(f_{\tau}\right)\right) .
$$

Lemma 4.2 thus implies $\frac{\sigma}{\sigma\left(f_{\tau}\right)} \sum_{j} \beta_{j} \leq 1$.

Proof of Theorem 4.1. - Since $(1 / \sigma, \ldots, 1 / \sigma)$ lies in the interior of $F_{0}(f)$, by convexity one can write

$$
(1 / \sigma, \ldots, 1 / \sigma)=\sum_{i} \alpha_{i} P_{i}+\sum_{j} \beta_{j} R_{j}
$$

for some $\alpha_{i} \geq 0$ and $\beta_{j} \geq 0$ with $\sum_{i} \alpha_{i}+\sum_{j} \beta_{j}=1$ and with $P_{i}$ finitely many integral points of $F_{0}(f) \backslash \tau$ and $R_{j}$ finitely many integral points of $\tau$. For $k \in \mathbf{N}^{k}$ with $F(f)(k)=\tau$ calculate

$$
\begin{aligned}
\nu(k) & =\sigma(1 / \sigma, \ldots, 1 / \sigma) \cdot k \\
& =\sigma\left(\sum_{i} \alpha_{i} P_{i}+\sum_{j} \beta_{j} R_{j}\right) \cdot k \\
& =\sigma\left(\sum_{i} \alpha_{i} P_{i} \cdot k+\sum_{j} \beta_{j} R_{j} \cdot k\right) \\
& \geq \sigma\left(\sum_{i} \alpha_{i}(N(k)+1)+\sum_{j} \beta_{j} N(k)\right) \\
& =\sigma\left(\left(\sum_{i} \alpha_{i}+\sum_{j} \beta_{j}\right)(N(k)+1)-\sum_{j} \beta_{j}\right) \\
& =\sigma\left((N(k)+1)-\sum_{j} \beta_{j}\right) \\
& \geq \sigma\left((N(k)+1)-\frac{\sigma\left(f_{\tau}\right)}{\sigma}\right)
\end{aligned}
$$


where (4.3.5) follows from $k \cdot R_{j}=N(k)$ and $k \cdot P_{i} \geq N(k)+1$ which is true by definition of $N(k)$, and where (4.3.8) follows from Corollary 4.3.

\section{Upper bounds for $A(p, m, \tau)$ and $B(p, m, \tau)$}

We recall one result from $[\mathbf{8}$.

5.1. Lemma ([8, Lemma (3.3)). - Let $C$ be a convex polyhedral cone in $\mathbf{R}_{+}^{n}$ generated by vectors in $\mathbf{N}^{n}$, and let $L$ be a linear form in $n$ variables with coefficients in $\mathbf{N}$. We denote by $C^{\text {int }}$ the interior of $C$ in the sense of Newton polyhedra. Let $\sigma>0$ and $\gamma \geq 0$ be real numbers satisfying

$$
\nu(k) \geq L(k) \sigma+\gamma, \text { for all } k \in C^{\text {int }} \cap \mathbf{N}^{n} .
$$

Put

$$
e=\operatorname{dim}\{k \in C \mid \nu(k)=L(k) \sigma\} .
$$

Then there exists a real number $c>0$ such that for all $m \in \mathbf{N}$ and for all $p \in \mathbf{R}$, with $p \geq 2$,

$$
\begin{aligned}
& \sum_{\substack{k \in C^{\text {int }} \cap \mathbf{N}^{n} \\
L(k)=m}} p^{-\nu(k)} \leq c p^{-m \sigma-\gamma}(m+1)^{\max (0, e-1)} . \\
& \\
& L(k)=m
\end{aligned}
$$

From this Lemma and from Theorem 4.1 follows:

5.2. Corollary. - Let $f, A(p, m, \tau)$, and $B(p, m, \tau)$ be as in Proposition 3.1. Then there exists a real number $c>0$ such that for all integers $m>0$, for all faces $\tau$ of $\Delta_{0}(f)$, and for all big enough primes $p$

$$
A(p, m, \tau) \leq c p^{-m \sigma} m^{\kappa-1}
$$

and

$$
B(p, m, \tau) \leq c p^{-m \sigma+\sigma\left(f_{\tau}\right)} m^{\kappa-1}
$$

Proof. - To derive (5.2.1) from Lemma 5.1, note that $\nu(k) \geq N(k) \sigma$ for any $k \in$ $\mathbf{N}^{n}$, and that $\kappa=\operatorname{dim}\left\{k \in \mathbf{R}_{+}^{n} \mid \nu(k)=N(k) \sigma\right\} \geq 1$.

To derive (5.2.2) from Lemma 5.1 and Theorem 4.1, use for $C$ the topological closure of the convex hull of $\{0\} \cup\left\{k \in \mathbf{N}^{n} \mid F(k)=\tau\right\}$, and note that $C^{\text {int }} \cap \mathbf{N}^{n}=$ $\left\{k \in \mathbf{N}^{n} \mid F(k)=\tau\right\}$. Clearly $\kappa \geq 1$ and $\kappa \geq \operatorname{dim}\{k \in C \mid \nu(k)=N(k) \sigma\}$. By (4.1.1), $\nu(k) \geq N(k) \sigma+\sigma-\sigma\left(f_{\tau}\right)$ for all $k \in C^{\text {int }} \cap \mathbf{N}^{n}$. 


\section{Upper bounds for $\sigma(f)$ and $E\left(p, f_{\tau}\right)$}

By Theorem 4 of Katz [11], for $f(x)$ a nonconstant homogeneous polynomial in $n$ variables over $\mathbf{Z}$, and $d$ the dimension of $\operatorname{grad} f=0$ in $\mathbf{A}_{\mathbf{C}}^{n}$, there exists $c$ such that for all big enough $p$ one has

$$
\left|\sum_{x \in \mathbf{A}^{n}\left(\mathbf{F}_{p}\right)} \exp \left(\frac{2 \pi i}{p} f(x)\right)\right| \leq c p^{\frac{n+d}{2}} .
$$

Moreover, $c$ can be taken depending on the degree of $f$ only. This implies:

6.1. Corollary. - Suppose that $f(x)$ is homogeneous of degree $\geq 2$ and let $d$ be the dimension of $\operatorname{grad} f=0$ in $\mathbf{A}_{\mathbf{C}}^{n}$. Then there exists $c$ such that for all $p$ big enough

$$
\left|\sum_{x \in \mathbf{G}_{m}^{n}\left(\mathbf{F}_{p}\right)} \exp \left(\frac{2 \pi i}{p} f(x)\right)\right|<c p^{\frac{n+d}{2}}
$$

and hence, for some $c^{\prime}$ one has, for all big enough $p$,

$$
|E(p, f)|<c^{\prime} p^{\frac{-n+d}{2}}
$$

with $E(p, f)$ as defined by (3.1.2). Moreover, $c$ and $c^{\prime}$ can be taken depending on $\Delta_{0}(f)$ only.

Proof. - Let $f_{0}\left(x_{2}, \ldots, x_{n}\right)$ be the polynomial $f\left(0, x_{2}, \ldots, x_{n}\right)$. Clearly $f_{0}$ is homogeneous in $n-1$ variables. By Katz' result (6.0.3) it is enough to show that $n-1+d\left(f_{0}\right) \leq n+d$, with $d\left(f_{0}\right)$ the dimension of $\operatorname{grad} f_{0}=0$ in $\mathbf{A}_{\mathbf{C}}^{n-1}$. This inequality follows from writing

$$
f(x)=x_{1} g(x)+f_{0}\left(x_{2}, \ldots, x_{n}\right)
$$

with $g$ a polynomial in $x$, and comparing $\operatorname{grad} f$ with $\operatorname{grad} f_{0}$.

6.2. - Let $\left\{\left(N_{i}, \nu_{i}\right)\right\}_{i \in I}$ be the numerical data of a resolution $h$ of $f$ with normal crossings (that is, if $\pi_{f}: Y \rightarrow \mathbf{A}_{\mathbf{C}}^{n}$ is an embedded resolution of singularities with normal crossings of $f=0$, then, for each irreducible component $E_{i}$ of $\pi_{f}^{-1} \circ f^{-1}(0)$, $i \in I$, let $N_{i}$ be the multiplicity of $E_{i}$ in $\operatorname{div}\left(f \circ \pi_{f}\right)$, and $\nu_{i}-1$ the multiplicity of $E_{i}$ in the divisor associated to $\pi_{f}^{*}\left(d x_{1} \wedge \ldots \wedge d x_{n}\right)$, cf. [3]). The essential numerical data of $\pi_{f}$ are the pairs $\left(N_{i}, \nu_{i}\right)$ for $i \in J$ with $J=I \backslash I^{\prime}$ and where $I^{\prime}$ is the set of indices $i$ in $I$ such that $\left(N_{i}, \nu_{i}\right)=(1,1)$ and such that $E_{i}$ does not intersect another $E_{j}$ with $\left(N_{j}, \nu_{j}\right)=(1,1)$. Define $\alpha\left(\pi_{f}\right)$ as

$$
\alpha\left(\pi_{f}\right)=-\min _{i \in J} \frac{\nu_{i}}{N_{i}}
$$

when $J$ is nonempty and define $\alpha\left(\pi_{f}\right)$ as $-2 n$ otherwise.

It follows from [2], Theorem 5.1 and Corollary 3.4, that

$$
\alpha\left(\pi_{f}\right) \geq \frac{-n+d}{2},
$$


with $d$ the dimension of $\operatorname{grad} f=0$ in $\mathbf{A}_{\mathbf{C}}^{n}$, and where the empty scheme has dimension $-\infty$.

6.3. Lemma. - Let $f$ be homogeneous of degree $\geq 2$ and nondegenerate w.r.t. the faces of $\Delta_{0}(f)$. Let $d$ be the dimension of $\operatorname{grad} f=0$ in $\mathbf{A}_{\mathbf{C}}^{n}$. Then

$$
\sigma(f) \leq \frac{n-d}{2}
$$

Proof. - By properties of a toric resolution $\pi_{f}$ of $f=0$, one has that

$$
\sigma(f)=-\alpha\left(\pi_{f}\right)
$$

with $\alpha\left(\pi_{f}\right)$ as defined by (6.2.1). Now use (6.2.2).

From (6.3.1) and Corollary 6.1, applied to $f_{\tau}$, follows:

6.4. Corollary. - Let $f$ be a homogeneous polynomial of degree $\geq 2$ which is nondegenerate w.r.t. the faces of $\Delta_{0}(f)$. Then there exists c such that for all faces $\tau$ of $\Delta_{0}(f)$ and all big enough primes $p$

$$
\left|E\left(p, f_{\tau}\right)\right|<c p^{-\sigma\left(f_{\tau}\right)}
$$

with $E\left(p, f_{\tau}\right)$ as defined by (3.1.2). Moreover, c can be taken depending on $\Delta_{0}(f)$ only.

\section{Proof of the main theorem}

Proof of Theorem 2.1. - When the degree of $f$ is $\geq 2$, use Proposition 3.1, Corollary 5.2, and (6.4.1). For linear $f$ the theorem is trivial.

\section{Comparison with the Denef-Sperber approach}

As mentioned above, Denef and Sperber [8] prove Theorem 2.1] under the extra condition that no vertex of $F_{0}(f)$ belongs to $\{0,1\}^{n}$. Key points in our proof of Theorem 2.1 are (4.1.1) (which implies Corollary 5.2) and (6.4.1). Instead of (4.1.1), Denef and Sperber used their result that, for similar $k$ as in (4.1.1) but assuming the extra condition that no vertex of $F_{0}(f)$ belongs to $\{0,1\}^{n}$,

$$
\nu(k) \geq \sigma(f)(N(f)(k)+1)-\frac{\operatorname{dim} \tau+1}{2} .
$$

This often fails if one omits the extra condition, see Examples (1) and (2) below. Instead of (6.4.1), they used the Adolphson-Sperber [1], Denef-Loeser [4] bounds

$$
\left|E\left(p, f_{\tau}\right)\right|<c p^{\frac{-\operatorname{dim} \tau-1}{2}}
$$


which hold (in particular) under the same conditions as for (6.4.1), but which are sometimes not as good as the bounds (6.4.1) (2)

We give two examples where our methods really make a difference with (8.0.2) and $(8.0 .3)$.

\section{Examples.}

(1) First, for $f(x, y, z, u)=x y+z u$ and $\tau=F_{0}(f)$, one has $\operatorname{dim} \tau=1, \sigma\left(f_{\tau}\right)=$ $\sigma=2$, 8.0.2) does not hold and (8.0.3) is not optimal, while (6.4.1) yields the optimal $\left|E\left(p, f_{\tau}\right)\right|<c p^{-2}$.

(2) Secondly, for $f(x, y, z, u)=x y+z u+x z+a y u$ with $a \in \mathbf{Z}, a \neq 1$, and $\tau=F_{0}(f)$, one has $\operatorname{dim} \tau=2, \sigma\left(f_{\tau}\right)=\sigma=2$, (8.0.2) does not hold and (8.0.3) is not optimal, while (6.4.1) yields again the optimal $\left|E\left(p, f_{\tau}\right)\right|<c p^{-2}$ for big $p$. In this example, $E\left(p, f_{\tau}\right)$ can be calculated by performing a transformation on $\mathbf{G}_{m}^{4}$ coming from an element of $G L_{n}(\mathbf{Z})$ transforming $f(x)$ into $f\left(x^{\prime}, y^{\prime}, z^{\prime}, u^{\prime}\right)=$ $x^{\prime}+y^{\prime}+z^{\prime}+a x^{\prime} y^{\prime} z^{\prime-1}$; the bounds for $E\left(p, f_{\tau}\right)$ are surprisingly sharp compared, for example, to bounds for the resembling Kloosterman sums.

\section{Analogues over finite extensions of $\mathbf{Q}_{p}$ and over $\mathbf{F}_{q}((t))$}

For any nonarchimedean local field $K$ with valuation ring $\mathcal{O}_{K}$, write $\psi_{K}$ for an additive character

$$
\psi_{K}: K \rightarrow \mathbf{C}^{\times}
$$

that is trivial on $\mathcal{O}_{K}$ but nontrivial on some element of $K$ of order -1 . Write $\operatorname{ord}_{K}: K^{\times} \rightarrow \mathbf{Z}$ for the valuation, $|\cdot|_{K}: K \rightarrow \mathbf{R}$ for the norm on $K$, and $\bar{K}$ for its residue field, with $q_{K}$ elements. Let $k$ be a number field with ring of integers $\mathcal{O}_{k}$. In this section $f$ is a nonconstant polynomial over $\mathcal{O}_{k}[1 / N]$ in $n$ variables, with $f(0)=0$ and $N \in \mathbf{Z}$. For $K$ any nonarchimedean local field that is an algebra over $\mathcal{O}_{k}[1 / N]$ and for $y \in K^{\times}$, consider the exponential integral

$$
S_{f, K}(y):=\int_{\mathcal{O}_{K}^{n}} \psi_{K}(y f(x))|d x|_{K},
$$

with $|d x|_{K}$ the normalized Haar measure on $K^{n}$. Note that $K$ may be of positive characteristic. Then the following generalization of Theorem 2.1 holds:

9.1. Theorem. - Suppose that $f$ is a homogeneous polynomial over $\mathcal{O}_{k}[1 / N]$ which is nondegenerate w.r.t. the faces of $\Delta_{0}(f)$. Then there exist $c>0$ and $M>N$ such that

$$
\left|S_{f, K}(y)\right| \leq c|y|_{K}^{-\sigma}\left|\operatorname{ord}_{K}(y)\right|^{\kappa-1}
$$

for all nonarchimedean local fields $K$ that are algebras over $\mathcal{O}_{k}[1 / N]$ and have residue characteristic $>M$, and all $y \in K^{\times}$with $\operatorname{ord}_{K}(y)<0$, with $|\cdot|$ the complex norm. Moreover, $c$ can be taken depending on $\Delta_{0}(f)$ only.

${ }^{(2)}$ Although (8.0.3) is sometimes sharper than (6.4.1) in cases where it does not matter for our course. 
Proof. - Same proof as of Theorem 2.1, using Proposition 9.2 instead of Proposition 3.1 .

9.2. Proposition. - Suppose that $f$ is nondegenerate w.r.t. (all) the faces of $\Delta_{0}(f)$. Then there exists $M>N$ such that

$$
S_{f, K}(y)=\left(1-q_{K}^{-1}\right)^{n} \sum_{\tau \text { face of } \Delta_{0}(f)}\left(A\left(q_{K}, m, \tau\right)+E(\bar{K}, \tau, y) B\left(q_{K}, m, \tau\right)\right)
$$

for all nonarchimedean local fields $K$ that are algebras over $\mathcal{O}_{k}[1 / N]$ and have residue characteristic $>M$ and all $y \in K^{\times}$with $\operatorname{ord}_{K}(y) \leq 0 .(3)$

In these formulas, $A\left(q_{K}, m, \tau\right)$ and $B\left(q_{K}, m, \tau\right)$ are as in Proposition [3.1, and

$$
E(\bar{K}, \tau, y):=\frac{1}{\left(q_{K}-1\right)^{n}} \sum_{u \in \mathbf{G}_{m}^{n}(\bar{K})} \psi_{y}\left(f_{\tau}(u)\right),
$$

with $\psi_{y}$ a nontrivial additive character on $\bar{K}$ depending on $y$ and $\psi_{K}(4)$

Proof. - Same proof as of Proposition 3.1.

Acknowledgment. - I would like to thank J. Denef, E. Hrushovski, and F. Loeser for inspiring discussions during the preparation of this paper.

\section{References}

[1] A. Adolphson and S. Sperber, Exponential sums and Newton polyhedra: cohomology and estimates, Ann. of Math. 130 (1989), no. 2, 367-406.

[2] R. Cluckers, Igusa's conjecture on exponential sums modulo $p$ and $p^{2}$ and the motivic oscillation index, to appear in Int. Math. Res. Not. IMRN, arXiv:math.NT/0602438.

[3] J. Denef, Report on Igusa's local zeta function, Séminaire Bourbaki Vol. 1990/91, Exp. No.730-744 (1991), 359-386, Astérisque 201-203, http://wis.kuleuven.be/algebra/denef.html ઔD2.

[4] J. Denef and F. Loeser, Weights of exponential sums, intersection cohomology, and Newton polyhedra, Invent. Math. 106 (1991), no. 2, 275-294.

[5] J. Denef and P. Sargos, Polyèdre de Newton et distribution $f_{+}^{s}$. I, J. Analyse Math. 53 (1989), 201-218, (French) [The Newton polyhedron and the distribution $f_{+}^{s}$. I].

[6] _ Polyèdre de Newton et distribution $f_{+}^{s}$. II, Math. Ann. 293 (1992), no. 2.

[7] J. Denef and S. Sperber, Exponential sums $\bmod p^{n}$ and Newton polyhedra, Manuscript (1990).

[8] _ Exponential sums mod $p^{n}$ and Newton polyhedra, Bull. Belg. Math. Soc. Simon Stevin suppl. (2001), 55-63.

[9] J. Denef and W. Veys, On the holomorphy conjecture for Igusa's local zeta function, Proc. Amer. Math. Soc. 123 (1995), no. 10, 2981-2988.

$\overline{{ }^{(3)} \text { For such } K}$ and for $y \in K^{\times}$with $\operatorname{ord}_{K}(y) \geq 0$, one has $S_{f, K}(y)=1$.

${ }^{(4)}$ In fact, the character $\psi_{y}$ only depends on $\psi_{K}$ and on $\overline{\mathrm{ac}}(y)$ for any multiplicative homomorphism $\overline{\mathrm{ac}}: K^{\times} \rightarrow \bar{K}^{\times}$extending the natural projection $\mathcal{O}_{K}^{\times} \rightarrow \bar{K}^{\times}$. 
[10] J. Igusa, Lectures on forms of higher degree (notes by S. Raghavan), Lectures on mathematics and physics, Tata institute of fundamental research, vol. 59, Springer-Verlag, 1978.

[11] N. Katz, Estimates for "singular" exponential sums, Int. Math. Res. Not. IMRN (1999), no. 16, 875-899.

Raf Cluckers, Katholieke Universiteit Leuven, Departement wiskunde, Celestijnenlaan 200B, B-3001 Leuven, Belgium. Current address: École Normale Supérieure, Département de mathématiques et applications, 45 rue d'Ulm, 75230 Paris Cedex 05, France

E-mail : raf.cluckers@wis.kuleuven.be • Url : www.dma.ens.fr/ cluckers/ 